\title{
Moment Problems on Bounded and Unbounded Domains
}

\author{
Octav Olteanu \\ Department of Mathematics-Informatics, Faculty of Applied Sciences, University Politehnica of Bucharest, 060042 Bucharest, Romania
}

Correspondence should be addressed to Octav Olteanu; olteanuoctav@yahoo.ie

Received 14 September 2012; Accepted 29 November 2012

Academic Editor: Zhijun Qiao

Copyright (C) 2013 Octav Olteanu. This is an open access article distributed under the Creative Commons Attribution License, which permits unrestricted use, distribution, and reproduction in any medium, provided the original work is properly cited.

Using approximation results, we characterize the existence of the solution for a two-dimensional moment problem in the first quadrant, in terms of quadratic forms, similar to the one-dimensional case. For the bounded domain case, one considers a space of complex analytic functions in a disk and a space of continuous functions on a compact interval. The latter result seems to give sufficient (and necessary) conditions for the existence of a multiplicative solution.

\section{Introduction}

Applying the extension Hahn-Banach type results in existence questions concerning the moment problem is a wellknown technique [1-11]. One of the most useful results is lemma of the majorizing subspace (see [12, Section 5.1.2] for the proof of the lattice-version of this lemma; see also [13]). It says that if $f$ is a linear positive operator on a subspace $S$ of the ordered vector space $X$, the target space being an order complete vector lattice $Y$, and for each $x \in X$ there is $s \in S, x \leq s$, then $f$ has a linear positive extension $F$ : $X \rightarrow Y$. Another geometric remark is that in the real case, the sublinear functional from the Hahn-Banach theorem can be replaced by a convex one. The theorem remains valid when the convex dominating functional is defined on a convex subset $A$ with some qualities with respect to the subspace $S$ (for instance, $S \cap \operatorname{ri}(A) \neq \Phi)$, where $\operatorname{ri}(A)$ is the relative interior of $A$ ). Here we recall an answer published without proof in 1978 [14], without losing convexity, but strongly generalizing the classical result. The first detailed proof was published in 1983 [15]. The proof of a similar result, in terms of the moment problem, was published in [10]. Here we recall the general statement from [14]. One of the reasons is that many other results are consequences of this theorem, including Bauer's theorem [13], Namiokas's theorem, and abstract moment problem-results published firstly in [9]. Part of these generalizations of the Hahn-Banach principle are applied in the present work too. Throughout this first part, $X$ will be a real vector space, $Y$ an order-complete vector lattice,
$A, B \subset X$ convex subsets, $q: A \rightarrow Y$ a concave operator, $p: B \rightarrow Y$ a convex operator, $S \subset X$ a vector subspace, and $f: S \rightarrow Y$ a linear operator.

Theorem 1. Assume that

$$
\left.f\right|_{S \cap A} \geq\left. q\right|_{S \cap A},\left.\quad f\right|_{S \cap B} \leq\left. p\right|_{S \cap B} .
$$

The following assertions are equivalent:

(a) there is a linear extension $F: X \rightarrow Y$ of the operator $f$ such that

$$
\left.F\right|_{A} \geq q,\left.\quad F\right|_{B} \leq p ;
$$

(b) there are $p_{1}: A \rightarrow Y$ convex and $q_{1}: B \rightarrow Y$ concave operators such that for all

$$
\left(\rho, t, \lambda^{\prime}, a_{1}, a^{\prime}, b_{1}, b^{\prime}, v\right) \in[0,1]^{2} \times(0, \infty) \times A^{2} \times B^{2} \times S,
$$

one has

$$
\begin{aligned}
(1-t) a_{1}-t b_{1} & =v+\lambda^{\prime}\left[(1-\rho) a^{\prime}-\rho b^{\prime}\right] \\
& \Longrightarrow(1-t) p_{1}\left(a_{1}\right)-t q_{1}\left(b_{1}\right) \\
& \geq f(v)+\lambda^{\prime}\left[(1-\rho) q\left(a^{\prime}\right)-\rho p\left(b^{\prime}\right)\right] .
\end{aligned}
$$

The minus-sign appears to construct a convex operator in the left-hand side member and a concave operator in 
the right side. The idea of sandwich theorem on arbitrary convex subsets $A, B$ is clear. Most of the applications hold for linear positive operators on linear ordered spaces $\left(X, X_{+}\right)$, when we take $A=X_{+}, q \equiv 0, B=X, p$ a suitable convex operator (a vector-valued norm, a sublinear operator), which "measures the continuity" of the extension F. One obtains the following result related to the theorem of H. Bauer ([13, Section 5.4]).

Theorem 2. Let $X$ be a preordered vector space with its positive cone $X_{+}, p: X \rightarrow Y$ a convex operator, $S \subset X$ a vector subspace, $f: S \rightarrow Y$ a linear positive operator. The following assertions are equivalent:

(a) there is a linear positive extension $F: X \rightarrow Y$ of $f$ such that $F(x) \leq p(x)$ for all $x \in X$;

(b) $f\left(x^{\prime}\right) \leq p(x)$ for all $\left(x^{\prime}, x\right) \in S \times X$ such that $x^{\prime} \leq x$.

Now we can deduce the main results on the abstract moment problem [9].

Theorem 3. Let $X, Y, p: X \rightarrow Y$ be as in Theorem 2, $\left\{x_{j}\right\}_{j \in J} \subset X,\left\{y_{j}\right\}_{j \in J} \subset Y$ given families. The following assertions are equivalent:

(a) there is a linear positive operator $F: X \rightarrow Y$ such that

$F\left(x_{j}\right)=y_{j} \quad \forall j \in J, \quad F(x) \leq p(x) \quad \forall x \in X$

(b) for any finite subset $J_{0} \subset J$ and any $\left\{\lambda_{j}\right\}_{j \in J_{0}} \subset R$, one has:

$$
\sum_{j \in J_{0}} \lambda_{j} x_{j} \leq x \Longrightarrow \sum_{j \in J_{0}} \lambda_{j} y_{j} \leq p(x) .
$$

A clearer sandwich-moment problem variant is the following one.

Theorem 4. Let $X, Y,\left\{x_{j}\right\}_{j \in J},\left\{y_{j}\right\}_{j \in J}$ be as in Theorem 3 and $F_{1}, F_{2} \in L(X, Y)$ two linear operators. The following statements are equivalent:

(a) there is a linear operator $F \in L(X, Y)$ such that

$$
\begin{gathered}
F_{1}(x) \leq F(x) \leq F_{2}(x) \quad \forall x \in X_{+}, \\
F\left(x_{j}\right)=y_{j}, \quad \forall j \in J
\end{gathered}
$$

(b) for any finite subset $J_{0} \subset J$ and any $\left\{\lambda_{j}\right\}_{j \in J_{0}} \subset R$, one has:

$$
\begin{gathered}
\left(\sum_{j \in J_{0}} \lambda_{j} x_{j}=\varphi_{2}-\varphi_{1}, \varphi_{1}, \varphi_{2} \in X_{+}\right) \\
\Longrightarrow \sum_{j \in J_{0}} \lambda_{j} y_{j} \leq F_{2}\left(\varphi_{2}\right)-F_{1}\left(\varphi_{1}\right) .
\end{gathered}
$$

We recall the following approximation lemma on an unbounded bi-dimensional subset.
Lemma 5 (see [7] and [8, Lemma 1.3(d)]). If $x \in C_{0}([0, \infty) \times$ $[0, \infty))$ is a nonnegative continuous function with compact support, then there exists a sequence $\left(p_{m}\right)_{m}$ of positive polynomials on $[0, \infty) \times[0, \infty)$, such that

$$
p_{m}(t)>x(t) \quad \forall t \geq 0, \forall m \in Z_{+}, p_{m} \longrightarrow x,
$$

uniformly on compact subsets of $[0, \infty) \times[0, \infty)$.

The idea of the proof is to add the $\infty$ point and to apply the Stone-Weierstrass theorem to the subalgebra generated by the functions $\exp \left(-m t_{1}-n t_{2}\right), m, n \in Z_{+}$. Then one uses for each such exp-function suitable majorizing or minorizing partial sums polynomials.

Note that Lemma 1.4 [8] asserts the density of positive polynomials in $\left(L_{v}^{1}(A)\right)_{+}$, for any closed subset $A$ of a finite dimensional space, $v$ being a positive regular Borel $\mathrm{M}$ determinate measure. The results of this work are generally applications of the theorems stated above.

For uniqueness of the solution see [1, 3, 4, 16-19]. Several other interesting related results are contained in [20] (application of fixed-point principle, iterative methods) [6], (construction of a solution), [11,21].

\section{Moment Problems on Unbounded Subsets}

The first result of this section concerns the multidimensional moment problem, being a consequence of the Stone Weierstrass theorem and of other usual results on positive polynomials on $[0, \infty)$. It is known that in several dimensions there are positive polynomials on $R_{+}^{n}$ which cannot be written involving sums of squares, as in the one-dimensional case. However, the approximation results from below seem to show that we can work with limits of tensor products of polynomials of one variable, for which such representation hold in each variable. This leads to characterizations of the existence of the solutions for multidimensional moment problems in terms of quadratic forms (Theorem 6(b)). Let $H$ be a Hilbert space, $A_{1}, A_{2}$ two positive commuting selfadjoint operators acting on $H$, with spectrums $\sigma\left(A_{j}\right), j=$ 1,2 . We introduce the commutative algebra $Y=Y\left(A_{1}, A_{2}\right)$ of self-adjoint operators $[5,12]$, which is also an order-complete vector lattice:

$$
\begin{gathered}
Y_{1}=\left\{T \in \mathrm{A}(H) ; T A_{j}=A_{j} T, j=1,2\right\}, \\
Y=\left\{U \in Y_{1} ; U T=T U \forall T \in Y_{1}\right\} .
\end{gathered}
$$

We denote by $X$ the space of all continuous functions $x$ : $[0, \infty)^{2} \rightarrow R$, with the modulus dominated by a polynomial at each point of $[0, \infty)^{2}$, and by $x_{(j, k)}$ the elements of the base of polynomials, namely, $x_{(j, k)}\left(t_{1}, t_{2}\right)=t_{1}^{j} t_{2}^{k}, t_{j} \geq 0$. 
Theorem 6 (see also [8, Theorem 3.2]). Let $\left(B_{(j, k)}\right)_{(j, k) \in Z_{+}^{2}} \subset$ $Y$. The following assertions are equivalent:

(a) there is a linear operator $F \in L(X, Y)$ such that

$$
\begin{gathered}
F\left(x_{(j, k)}\right)=B_{(j, k)} \quad \forall(j, k) \in Z_{+}^{2}, \\
0 \leq F(x) \leq \int_{\sigma\left(A_{1}\right) \times \sigma\left(A_{2}\right)} x\left(t_{1}, t_{2}\right) d E_{A_{1}} d E_{A_{2}} \quad \forall x \in X_{+}, \\
|F(\varphi)| \leq \int_{\sigma\left(A_{1}\right) \times \sigma\left(A_{2}\right)}\left|\varphi\left(t_{1}, t_{2}\right)\right| d E_{A_{1}} d E_{A_{2}} \quad \forall \varphi \in X ;
\end{gathered}
$$

(b) for any finite subsets $J_{1}, J_{2} \subset Z_{+}$and any $\left\{\alpha_{j}\right\}_{j \in J_{1}}$, $\left\{\beta_{k}\right\}_{k \in J_{2}}$, one has:

$$
\begin{aligned}
0 & \leq \sum_{i, j \in J_{1}, k, l \in J_{2}} \alpha_{i} \alpha_{j} \beta_{k} \beta_{l} B_{(i+j, k+l)} \\
& \leq \sum_{i, j \in J_{1}, k, l \in J_{2}} \alpha_{i} \alpha_{j} \beta_{k} \beta_{l} A_{1}^{i+j} A_{2}^{k+l}, \\
0 & \leq \sum_{i, j \in J_{1}, k, l \in J_{2}} \alpha_{i} \alpha_{j} \beta_{k} \beta_{l} B_{(i+j+1, k+l)} \\
& \leq \sum_{i, j \in J_{1}, k, l \in J_{2}} \alpha_{i} \alpha_{j} \beta_{k} \beta_{l} A_{1}^{i+j+1} A_{2}^{k+l}, \\
0 & \leq \sum_{i, j \in J_{1}, k, l \in J_{2}} \alpha_{i} \alpha_{j} \beta_{k} \beta_{l} B_{(i+j, k+l+1)} \\
& \leq \sum_{i, j \in J_{1}, k, l \in J_{2}} \alpha_{i} \alpha_{j} \beta_{k} \beta_{l} A_{1}^{i+j} A_{2}^{k+l+1}, \\
0 & \leq \sum_{i, j \in J_{1}, k, l \in J_{2}} \alpha_{i} \alpha_{j} \beta_{k} \beta_{l} B_{(i+j+1, k+l+1)} \\
& \leq \sum_{i, j \in J_{1}, k, l \in J_{2}} \alpha_{i} \alpha_{j} \beta_{k} \beta_{l} A_{1}^{i+j+1} A_{2}^{k+l+1} .
\end{aligned}
$$

Proof. From the Stone-Weierstrass theorem, we can infer that the space $S=C_{0}\left(\sigma\left(A_{1}\right)\right) \otimes C_{0}\left(\sigma\left(A_{2}\right)\right)$ is dense in the space $C_{0}\left(\sigma\left(A_{1}\right) \times \sigma\left(A_{2}\right)\right)$. It follows that any continuous nonnegative function $x$ on the product of spectrums can be uniformly approximated from above with elements of $S$. We extend all the functions involved with zero outside the compacts where they were defined, using then Luzin's theorem. Thus we obtain positive continuous approximations with compact support of $x$, defined on $[0, \infty)^{2}$. Each such approximation is the limit of a sequence of positive functions from $C_{0}([0, \infty)) \otimes C_{0}([0, \infty))$. Let $s=x_{1} \otimes x_{2}$ be such a function. For each of the separate variables $t_{1}, t_{2}$, there is a sequence of positive polynomials on the whole nonnegative semi-axes such that

$$
p_{m, j}>x_{j} \geq 0, \quad \forall m \in Z_{+}, p_{m, j} \longrightarrow x_{j}, m \longrightarrow \infty, j=1,2
$$

the convergence being uniform on compact subsets. Because the polynomials involved are positive on the whole interval $[0, \infty)$, from $[1]$ we know their form:

$$
p_{m, j}\left(t_{j}\right)=q_{m, j}^{2}\left(t_{j}\right)+t_{j} r_{m, j}^{2}\left(t_{j}\right), \quad j=1,2, m \in Z_{+} .
$$

We define a linear operator on the subspace $P_{1} \otimes P_{2}$ of products of polynomials in separate variables, such that the moment conditions from the statement holds:

$$
\begin{aligned}
f\left(\sum_{j, k} \alpha_{j} \beta_{k} t_{1}^{j} t_{2}^{k}\right) & =\sum_{j, k} \alpha_{j} \beta_{k} B_{(j, k)} \Longrightarrow f\left(x_{(j, k)}\right) \\
& =B_{(j, k)} \quad \forall(j, k) \in Z_{+}^{2} .
\end{aligned}
$$

We have already seen that these polynomials are dense in $C_{0}\left([0, \infty)^{2}\right)$. Using the above arguments, the assertion (b) says that we have

$$
\begin{array}{r}
0 \leq f\left(p_{1} \otimes p_{2}\right) \\
\leq \iint_{\sigma\left(A_{1}\right) \times \sigma\left(A_{2}\right)} p_{1}\left(t_{1}\right) p_{2}\left(t_{2}\right) d E_{A_{1}} d E_{A_{2}}, \\
\forall p_{j} \in\left(R\left[t_{j}\right]\right)_{+}, \quad j=1,2 .
\end{array}
$$

An application of the majorizing subspace lemma [12] leads to the existence of a positive linear extension $F: X \rightarrow Y$ of $f$. Using the uniform convergence of the special polynomials on the product of spectrums, we obtain

$$
\begin{aligned}
F(x) & =\lim F\left(p_{m, 1} \otimes p_{m, 2}\right) \\
& =\lim f\left(p_{m, 1} \otimes p_{m, 2}\right) \\
& \leq \lim \iint_{\sigma\left(A_{1}\right) \times \sigma\left(A_{2}\right)}\left(p_{m, 1} \otimes p_{m, 2}\right) d E_{A_{1}} d E_{A_{2}} \\
& =\iint_{\sigma\left(A_{1}\right) \times \sigma\left(A_{2}\right)} x\left(t_{1}, t_{2}\right) d E_{A_{1}} d E_{A_{2}}, \quad \forall x \in X_{+} .
\end{aligned}
$$

This relation leads to the last conclusion (a). Thus the proof of (b) $\Rightarrow$ (a) is finished. Since the converse is obvious, the theorem is proved.

Sometimes it is useful to find sufficient or necessary conditions for the existence of the solution in terms of some positive related sequences (assertion (b) of the next theorem).

Theorem 7. Let us denote $d v=\exp (-t) d t$ on $[0, \infty), X=$ $L_{v}^{1}=L_{v}^{1}([0, \infty))$, and let $\left(y_{j}\right)_{j=0}^{\infty}$ be a sequence of real numbers. The following assertions are equivalent:

(a) there exists a unique $g \in L_{v}^{\infty}([0, \infty))$ such that

$$
\begin{aligned}
& \int_{o}^{\infty} t^{j} g(t) \exp (-t) d t=y_{j} \\
& \forall j \in Z_{+}, 0 \leq g(t) \leq 1 \text { a.e.; }
\end{aligned}
$$


(b) $\sum_{j=0}^{n} \alpha_{j} t^{j} \geq 0$ for all $t \geq 0 \Rightarrow 0 \leq \sum_{j=0}^{n} \alpha_{j} y_{j} \leq$ $\sum_{j=0}^{n} \alpha_{j} \cdot \Gamma(j+1)$, for all $n \in Z_{+}$.

(c) for any finite family $\left(\lambda_{j}\right)_{j=0}^{n}$ of real numbers, one has

$$
\begin{aligned}
& 0 \leq \sum_{i, j=0}^{n} \lambda_{i} \lambda_{j} y_{i+j} \leq \sum_{i, j=0}^{n} \lambda_{i} \lambda_{j} \Gamma(i+j+1), \\
& 0 \leq \sum_{i, j=0}^{n} \lambda_{i} \lambda_{j} y_{i+j+1} \leq \sum_{i, j=0}^{n} \lambda_{i} \lambda_{j} \Gamma(i+j+2) .
\end{aligned}
$$

Proof $((b) \Rightarrow(a))$. Denote $x_{j}(t)=t^{j}, j \in Z_{+}$. One applies Theorem 4 , (b) $\Rightarrow$ (a). Let $n \in Z_{+}$and $\left(\lambda_{j}\right)_{j=0}^{n}$ in $R$ such that

$$
\begin{gathered}
\sum_{j=0}^{n} \lambda_{j} x_{j}=\varphi_{2}-\varphi_{1}, \quad \varphi_{l} \in X_{+} \bigcap C_{0}([0, \infty)) l=1,2, \\
X=\left\{x \in L_{v}^{1} ; \exists p \in P,|x| \leq p\right\}, P
\end{gathered}
$$

being the subspace of polynomials. From the approximation lemma mentioned in the end of the introduction (see [7, Lemma 1.4]), and using (b), we infer that:

$$
\begin{aligned}
\sum_{j=0}^{n} \lambda_{j} x_{j} & \leq p_{m}=\sum_{j=0}^{m} \beta_{j} x_{j} \longrightarrow \varphi_{2} \Longrightarrow f\left(\sum_{j=0}^{n} \lambda_{j} x_{j}\right):=\sum_{j=0}^{n} \lambda_{j} y_{j} \\
& \leq \sum_{j=0}^{m} \beta_{j} y_{j}=f\left(p_{m}\right) \leq \sum_{j=0}^{m} \beta_{j} \Gamma(j+1) \\
& \longrightarrow \int_{0}^{\infty} \varphi_{2}(t) \exp (-t) d t, \quad m \longrightarrow \infty .
\end{aligned}
$$

It follows that the linear form defined on polynomials is positive and dominated on $C_{0}([0, \infty))$ by the linear form $F_{2}$ defined below (note that $p_{m}$ are positive polynomials on $[0, \infty))$ :

$$
\begin{aligned}
F_{2}(\varphi) & :=\int_{0}^{\infty} \varphi(u) \exp (-u) d u, \\
F_{1} & \equiv 0 \Longrightarrow \sum_{j=0}^{n} \lambda_{j} y_{j} \leq \sum_{j=0}^{m} \beta_{j} y_{j} \\
& \longrightarrow \int_{0}^{\infty} \varphi_{2}(t) \exp (-t) d t \\
& =F_{2}\left(\varphi_{2}\right)-F_{1}\left(\varphi_{1}\right), \quad m \longrightarrow \infty .
\end{aligned}
$$

Application of Theorem 4 shows that there exists a linear positive form $F \in C_{0}^{*}$ satisfying the moment conditions and being majorized by $F_{2}$ on the positive cone $X_{+}$. Because of the density of $C_{c}([0, \infty)) \subset X$ in $L_{v}^{1}$, and of the continuity of $F_{2}$ with respect to $L_{v}^{1}$-norm, there is an extension
$\widetilde{F} \in\left(L_{v}^{1}\right)^{*} \cong L^{\infty}$ of $F$. It follows that there exists a function in $g \in L_{v}^{\infty}([0, \infty))$ representing $\widetilde{F}$ :

$$
\begin{aligned}
y_{j} & =f\left(x_{j}\right)=\widetilde{F}\left(x_{j}\right)=\int_{0}^{\infty} t^{j} g(t) d v \\
& =\int_{0}^{\infty} t^{j} g(t) \exp (-t) d t, \quad j \in Z_{+}, \\
0 & \leq \int_{0}^{\infty}(\varphi \cdot g)(t) \cdot \exp (-t) d t \\
& \leq \int_{0}^{\infty} \varphi(t) \cdot \exp (-t) d t, \quad \varphi \in X_{+} .
\end{aligned}
$$

For $\varphi=\chi_{E}, E$ Borel subset, one obtains

$$
\begin{array}{r}
0 \leq \int_{E} g(t) d v \leq \int_{E} d v=v(E) \\
\forall E \in \Sigma \Longrightarrow 0 \leq g \leq 1 \text { a.e. }
\end{array}
$$

Since a direct computation and application of the Carleman criterion [18] show that $d v=\exp (-t) d t$ is an M-determinate measure, it follows from the same criterion that $g \cdot d v$ is Mdeterminate too. Thus the proof of (a) $\Leftrightarrow$ (b) is finished. The equivalence (b) $\Leftrightarrow$ (c) follows easily by the aid of the form of positive polynomials on the nonnegative semi-axes.

Corollary 8. The set of all sequences $\left(y_{j}\right)_{j}$ verifying one of the assertions of Theorem 7 is a convex subset of $R^{Z_{+}}$, with a natural topology which makes it compact, and its extreme points are the sequences associated to those $g \in$ $U_{1}\left(L_{v}^{\infty}([0, \infty))\right) \cap\left(L_{v}^{\infty}([0, \infty))\right)_{+}$which take essentially only the values 0 and (or) 1 . For each extreme point $g$ with bounded support, one has

$$
\sum_{j=0}^{\infty} \frac{y_{j}}{j !}=m(\operatorname{Supp}(g))
$$

where $m$ denotes the Lebesgue measure.

\section{Moment Problems on Bounded Subsets}

The next statement concerns a space of complex analytic functions with real coefficients, considered as a real ordered vector space. We denote by $X_{+}$the convex cone of all absolutely convergent power series in the open disk $D=$ $\{|z|<b\}$, with continuous sum

$$
\sum_{j \in Z_{+}} \alpha_{j} z^{j}=\sum_{j \in Z_{+}} \alpha_{j} \varphi_{j}(z)
$$

in the closed disk $\bar{D}$, and with nonnegative coefficients. Let $X=X_{+}-X_{+}$. Let $\left(y_{j}\right)_{j \in Z_{+}}$be a sequence of real numbers. 
Theorem 9. If $a \in(0, b)$, consider the following assertions:

(a) there is a real linear functional F on $X$ such that

$$
\begin{aligned}
& F\left(\varphi_{j}\right)=y_{j}, \quad j \in Z_{+}, \\
& |F(\varphi)| \leq \frac{b}{b-a} \varphi(b), \quad \forall \varphi \in X_{+}
\end{aligned}
$$

(b) one has $\left|y_{j}\right| \leq a^{j}, j \in Z_{+}$.

Then (b) implies (a) holds.

Proof. One observes that for all $\varphi \in X_{+}$, we have $\|\varphi\|_{\infty}=$ $\varphi(b)=\varepsilon_{b}(\varphi)$. To apply Theorem 4 , let us consider the following relation and its derivates:

$$
\begin{aligned}
\sum_{j=0}^{n} \lambda_{j} \varphi_{j} & =\sum_{j=0}^{\infty} \alpha_{j} \varphi_{j}-\sum_{j=0}^{\infty} \beta_{j} \varphi_{j} \\
& =\sum_{j=0}^{\infty}\left(\alpha_{j}-\beta_{j}\right) \varphi_{j}, \quad \alpha_{j}, \beta_{j} \geq 0 .
\end{aligned}
$$

Identification of the corresponding coefficients, Cauchy's inequalities and the hypothesis (b) lead to

$$
\begin{aligned}
&-\frac{\varphi_{1}(b)}{b^{j}}=-\frac{\left\|\varphi_{1}\right\|_{\infty}}{b^{j}} \\
& \leq-\beta_{j} \leq \lambda_{j}=\alpha_{j}-\beta_{j} \leq \alpha_{j} \\
& \leq \frac{\left\|\varphi_{2}\right\|_{\infty}}{b^{j}}=\frac{\varphi_{2}(b)}{b^{j}}, \Longrightarrow \\
&\left|\lambda_{j}\right| \leq \frac{\varphi_{1}(b)+\varphi_{2}(b)}{b^{j}}, \quad j=0, \ldots, n \Longrightarrow \sum_{j=0}^{n} \lambda_{j} y_{j} \\
& \leq \sum_{j=0}^{n}\left|\lambda_{j}\right| \cdot\left|y_{j}\right| \leq\left(\varphi_{1}(b)+\varphi_{2}(b)\right)\left(\sum_{j=0}^{\infty} \frac{a^{j}}{b^{j}}\right) \\
&=\frac{b}{b-a} \varphi_{2}(b)-\left(-\frac{b}{b-a} \varphi_{1}(b)\right) \\
&=F_{2}\left(\varphi_{2}\right)-F_{1}\left(\varphi_{1}\right), \\
& F_{2}(\varphi):=\frac{b}{b-a} \varphi(b), \quad F_{1}=-F_{2} .
\end{aligned}
$$

Now from Theorem 4 we infer that there is a linear functional $F$ satisfying the moment conditions, such that

$$
-F_{2}(\varphi) \leq F(\varphi) \leq F_{2}(\varphi) \quad \forall \varphi \in X_{+} .
$$

The conclusion follows.

Remark 10. For fixed $0<a<b=1$, Theorem 9 gives an upper bound and a lower bound for the convex set of solutions, both of them being Dirac measures multiplied with a constant. These bounds are realized if and only if we have $y_{j}= \pm 1 /(1-a)$ for some $j \in Z_{+}$. The last results are applications of Theorem 3 . Let $T$ be a nonempty set, $X$ an algebra of functions on $T$ and $X_{+}$the cone of pointwise nonnegative functions on $T$. Let $Y$ be an order complete lattice, which is also a commutative algebra. Let

$$
\begin{array}{ll}
\left\{p_{\alpha}\right\}_{\alpha \in A} \subset X, \quad\left\{\varphi_{\beta}\right\}_{\beta \in B} \subset X, \\
\left\{u_{\alpha}\right\}_{\alpha \in A} \subset Y, \quad\left\{v_{\beta}\right\}_{\beta \in B} \subset Y
\end{array}
$$

be which $S=S p\left\{p_{\alpha} \varphi_{\beta}\right\}_{(\alpha, \beta) \in A \times B}$ is a majorizing subspace of $X$.

Theorem 11. The following assertions are equivalent:

(a) there exists a linear positive operator $F \in L_{+}(X, Y)$ such that

$$
\begin{aligned}
& F\left(p_{\alpha} \varphi_{\beta}\right)=u_{\alpha} v_{\beta} \quad \forall(\alpha, \beta) \in A \times B, \\
& x \leq \sum_{(\alpha, \beta)} \gamma_{(\alpha, \beta)} p_{\alpha} \varphi_{\beta} \Longrightarrow F(x) \leq \sum_{(\alpha, \beta)} \gamma_{(\alpha, \beta)} u_{\alpha} v_{\beta},
\end{aligned}
$$

where all the sums are finite;

(b) the following implication holds:

$$
\begin{aligned}
\sum_{(\alpha, \beta)} \lambda_{(\alpha, \beta)} p_{\alpha} \varphi_{\beta} & \leq \sum_{\left(\alpha^{\prime}, \beta^{\prime}\right)} \gamma_{\left(\alpha^{\prime}, \beta^{\prime}\right)} p_{\alpha^{\prime}} \varphi_{\beta^{\prime}} \\
& \Longrightarrow \sum_{(\alpha, \beta)} \lambda_{(\alpha, \beta)} u_{\alpha} v_{\beta} \\
& \leq \sum_{\left(\alpha^{\prime}, \beta^{\prime}\right)} \gamma_{\left(\alpha^{\prime}, \beta^{\prime}\right)} u_{\alpha^{\prime}} v_{\beta^{\prime}}
\end{aligned}
$$

Proof. The implication (a) $\Rightarrow(\mathrm{b})$ is obvious. For the converse observe that any element of $x \in X$ is majorized by an element from $S$. Writing this for $-x$ too, we get that any $x \in X$ is between two elements $s_{1}(x) \leq x \leq s_{2}(x), s_{j}(x) \in S, j=1,2$. We denote

$$
x_{(\alpha, \beta)}=p_{\alpha} \varphi_{\beta}, \quad y_{(\alpha, \beta)}=u_{\alpha} v_{\beta}, \quad(\alpha, \beta) \in A \times B .
$$

Define $p: X \rightarrow Y$,

$$
\begin{gathered}
p(x)=\inf \left\{\sum_{\left(\alpha, \beta^{\prime}\right)} \gamma_{\left(\alpha^{\prime} \cdot \beta^{\prime}\right)} u_{\alpha^{\prime}} v_{\beta^{\prime}} ;\right. \\
\left.x \leq \sum_{\left(\alpha, \beta^{\prime}\right)} \gamma_{\left(\alpha^{\prime} \cdot \beta^{\prime}\right)} p_{\alpha^{\prime}} \varphi_{\beta^{\prime}}\right\}, \quad x \in X .
\end{gathered}
$$

If $f: S \rightarrow Y$ is the linear operator verifying the moment conditions, then (b) says that $f$ is linear and positive on $S$, so that for any $s_{1} \leq x, s_{1} \in S$ we have

$$
f\left(s_{1}\right) \leq p(x) .
$$


It follows that $p$ is well defined and a standard computation shows that it is a convex operator. Moreover, these remarks and the definition of $p$ lead to the following implication:

$$
\begin{aligned}
\sum_{(\alpha, \beta)} \lambda_{(\alpha, \beta)} p_{\alpha} \varphi_{\beta} & =\sum_{(\alpha, \beta)} \lambda_{(\alpha, \beta)} x_{(\alpha, \beta)} \leq x \\
& \Longrightarrow \sum_{(\alpha, \beta)} \lambda_{(\alpha, \beta)} u_{\alpha} v_{\beta} \\
& =\sum_{(\alpha, \beta)} \lambda_{(\alpha, \beta)} y_{(\alpha, \beta)} \leq p(x)
\end{aligned}
$$

Hence the condition of Theorem 3, is accomplished, and the conclusion follows.

In the following result related to the preceding one, $Y$ will be as above; additionally assume that it is a Banach algebra with multiplication unit $u_{0}$, which is a strong order unit too. An example is the algebra of self-adjoint operators preceding Theorem 6 , when $u_{0}=I$. In the sequel, this will be the target algebra of our operators. Let

$$
\begin{aligned}
& X=C([a, b]), \quad p_{\alpha}(t)=\varphi_{\alpha}(t)=t^{\alpha}, \\
& \forall \alpha \in A=B=Z_{+}, \quad\left\{u_{\alpha}\right\}_{\alpha \in Z_{+}} \subset Y, \\
& \left\{v_{\alpha}\right\}_{\alpha \in Z_{+}} \subset Y, \quad u_{0}=v_{0}=I .
\end{aligned}
$$

Theorem 12. Under the above assumptions, the following assertions are equivalent:

(a) there is a nondecreasing mapping $\sigma:[a, b] \rightarrow Y$ such that:

$$
\begin{gathered}
\int_{a}^{b} t^{\alpha+\beta} d \sigma=u_{\alpha} v_{\beta} \forall(\alpha, \beta) \in Z_{+}^{2}, \\
x \leq \sum_{(\alpha, \beta)} \gamma_{(\alpha, \beta)} p_{\alpha+\beta} \\
\Longrightarrow \int_{a}^{b} x(t) d \sigma \leq \sum_{(\alpha, \beta)} \gamma_{(\alpha, \beta)} u_{\alpha} v_{\beta}, \\
\int_{a}^{b} x(t) \cdot y(t) d \sigma=\int_{a}^{b} x(t) d \sigma \\
\cdot \int_{a}^{b} y(t) d \sigma \forall x, \quad y \in C([a, b]),
\end{gathered}
$$

(b) the following implication holds:

$$
\begin{aligned}
\sum_{(\alpha, \beta)} \lambda_{(\alpha, \beta)} p_{\alpha+\beta} & \leq \sum_{\left(\alpha^{\prime}, \beta^{\prime \prime}\right)} \gamma_{\left(\alpha^{\prime}, \beta^{\prime}\right)} p_{\alpha^{\prime}+\beta^{\prime}} \\
& \Longrightarrow \sum_{(\alpha, \beta)} \lambda_{(\alpha, \beta)} u_{\alpha} v_{\beta} \leq \sum_{\left(\alpha^{\prime}, \beta^{\prime \prime}\right)} \gamma_{\left(\alpha^{\prime}, \beta^{\prime}\right)} u_{\alpha^{\prime}} v_{\beta^{\prime}} \\
u_{0}=v_{0} & =I .
\end{aligned}
$$

Proof. The implication (a) $\Rightarrow$ (b) is almost obvious: the linear operator defined by the vector measure $d \sigma$ is multiplicative and positive on $X$. To prove the converse, we use the preceding result of Theorem 11. There is a linear positive operator $F \in L_{+}(X, Y)$ such that

$$
\begin{gathered}
F\left(p_{\alpha} \varphi_{\beta}\right)=F\left(p_{\alpha+\beta}\right)=u_{\alpha} v_{\beta}, \quad \forall(\alpha, \beta) \in Z_{+}^{2}, \\
F(x) \leq p(x) \quad \forall x \in X .
\end{gathered}
$$

Since $F$ applies topological bounded subsets of $C([a, b])$ into order-bounded subsets of $Y$, the theorem in [12, page 272] asserts that there exists $\sigma$ representing mapping. Since $F$ is positive, $\sigma$ is nondecreasing. We have

$$
\begin{aligned}
F\left(p_{\alpha+\beta}\right) & =\int_{a}^{b} t^{\alpha+\beta} d \sigma=u_{\alpha} v_{\beta}=\left(u_{\alpha} v_{0}\right) \cdot\left(v_{\beta} v_{0}\right) \\
& =\int_{a}^{b} t^{\alpha} d \sigma \cdot \int_{a}^{b} t^{\beta} d \sigma \\
& =F\left(p_{\alpha}\right) \cdot F\left(p_{\beta}\right), \quad(\alpha, \beta) \in Z_{+}^{2} .
\end{aligned}
$$

The conclusion is that on basic monomials, $F$ is multiplicative. Now, a straightforward computation shows that $F$ is multiplicative on the subspace of polynomials. From the density of the latter in $C([a, b])$ and using also the continuity of the product operation on $Y \times Y$, we deduce that $F$ is multiplicative on the space of continuous functions. The proof is finished.

Corollary 13. If $F$ is the solution from Theorem 12, then denoting $B_{1}=F\left(u_{1}\right)=\int_{a}^{b} t d \sigma$, one must has

$$
F\left(p_{m}\right)=F\left(\varphi_{m}\right)=u_{m}=v_{m}=B_{1}^{m} \quad \forall m \in Z_{+}
$$

so that the measure do leads to the spectral measure of the operator $B_{1}$.

\section{References}

[1] N. I. Akhiezer, The Classical Moment Problem and Some Related Questions in Analysis, Oliver and Boyd, Edinburgh, UK, 1965.

[2] C. Ambrozie and O. Olteanu, "A sandwich theorem, the moment problem, finite-simplicial sets and some inequalities," Revue Roumaine de Mathématique Pures et Appliquées, vol. 49, pp. 189-210, 2004.

[3] G. Choquet, "Le problème des moments," in Séminaire d'Initiation à l'Analise, Institut H. Poicaré, Paris, France, 1962.

[4] M. G. Krein and A. A. Nudelman, Markov Moment Problem and Extremal Problems, American Mathematical Society, Providence, RI, USA, 1977.

[5] L. Lemnete-Ninulescu, "Using the solution of an abstract moment problem to solve some classical complex moment problems," Romanian Journal of Pure and Applied Mathematics, vol. 51, pp. 703-711, 2006.

[6] J. M. Mihăilă, O. Olteanu, and C. Udrişte, "Markov-type and operator-valued multidimensional moment problems, with some applications," Romanian Journal of Pure and Applied Mathematics, vol. 52, pp. 405-428, 2007. 
[7] J. M. Mihăilă, O. Olteanu, and C. Udrişte, "Markov-type moment problems for arbitrary compact and for some noncompact Borel subsets of $R^{n}$,' Romanian Journal of Pure and Applied Mathematics, vol. 52, pp. 655-664, 2007.

[8] A. Olteanu and O. Olteanu, "Some unexpected problems of the moment problem," in Proceedings of the 6th Congress of Romanian Mathematicians, vol. 1, pp. 347-355, Academiei, 2010.

[9] O. Olteanu, "Application de théorèmes de prolongement d'opérateurs linéaires au problème des moments et à une generalisation d'un théorème de Mazur-Orlicz,' Comptes Rendus de l'Académie des Sciences Série I, vol. 313, pp. 739-742, 1991.

[10] O. Olteanu, "Applications of a general sandwich theorem for operators to the moment problem," Revue Roumaine de Mathématique Pures et Appliquées, no. 2, pp. 513-521, 1996.

[11] O. Olteanu, "New aspects of the classical moment problem," Romanian Journal of Pure and Applied Mathematics, vol. 49, pp. 63-77, 2004.

[12] R. Cristescu, Ordered Vector Spaces and Linear Operators, Academiei, Bucharest and Abacus Press, Kent, UK, 1976.

[13] H. H. Schaefer, Topological Vector Spaces, Springer, New York, NY, USA, 1971.

[14] O. Olteanu, "Convexité et prolongement d’opérateurs linéaires," Comptes Rendus de l'Académie des Sciences, Série A, vol. 286, pp. 511-514, 1978.

[15] O. Olteanu, “Théorèmes de prolongement d’opérateurs linéaires," Revue Roumaine de Mathématique Pures et Appliquées, vol. 28, no. 10, pp. 953-983, 1983.

[16] C. Berg, J. P. R. Christensen, and C. U. Jensen, Harmonic Analysis on Semigroups: Theory of Positive Definite and Related Functions, Springer, New York, NY, USA, 1984.

[17] B. Fuglede, “The multidimensional moment problem," Expositiones Mathematicae, vol. 1, pp. 47-65, 1983.

[18] C. Kleiber and J. Stoyanov, "Multivariate distributions and the moment problem," Journal of Multivariate Analysis, vol. 113, pp. 7-18, 2013.

[19] J. Stoyanov, "Stieltjes classes for moment-indeterminate probability distributions," Journal of Applied Probability, vol. 41, no. 1, pp. 281-294, 2004.

[20] C. Berg and A. J. Durán, “The fixed point for a transformation of Hausdorff moment sequences and iteration of a rational function," Mathematica Scandinavica, vol. 103, no. 1, pp. 11-39, 2008.

[21] M. Putinar and F. H. Vasilescu, "Problème des moments sur les compacts semi-algébriques," Comptes Rendus de l'Académie des Sciences Série I, vol. 323, pp. 787-791, 1996. 


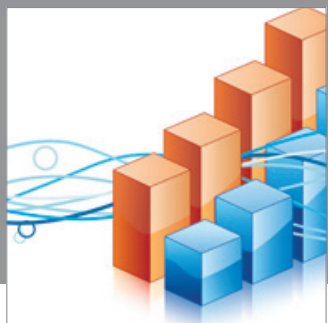

Advances in

Operations Research

mansans

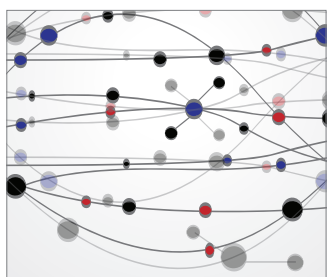

The Scientific World Journal
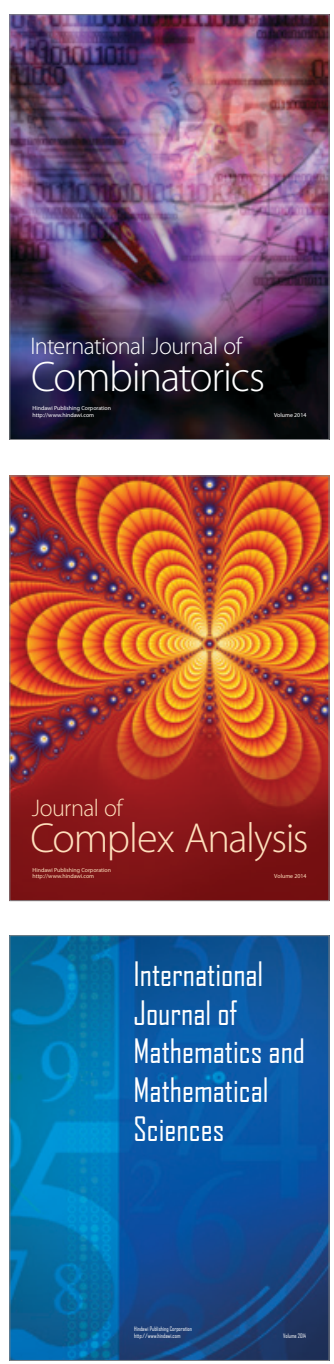
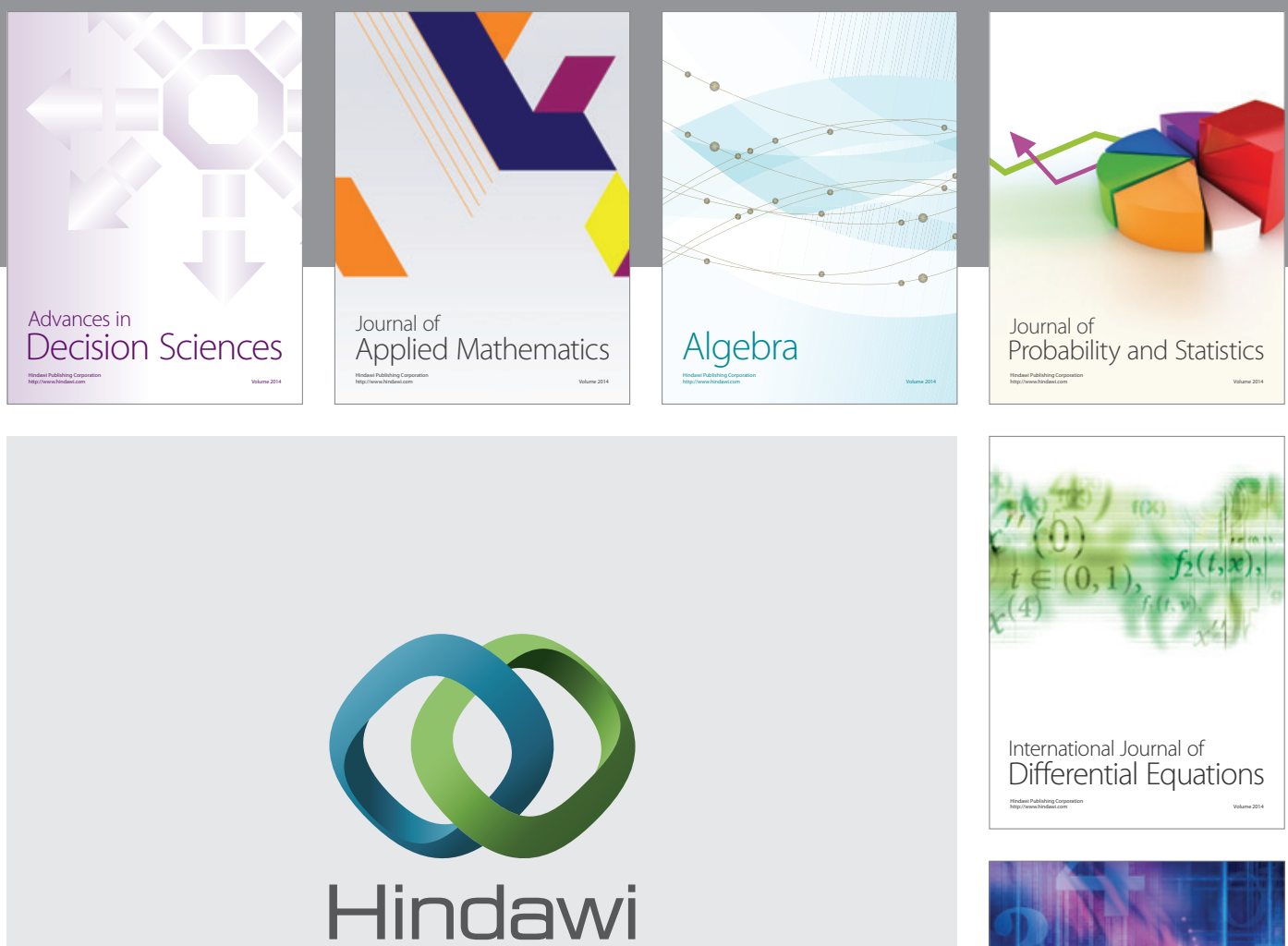

Submit your manuscripts at http://www.hindawi.com
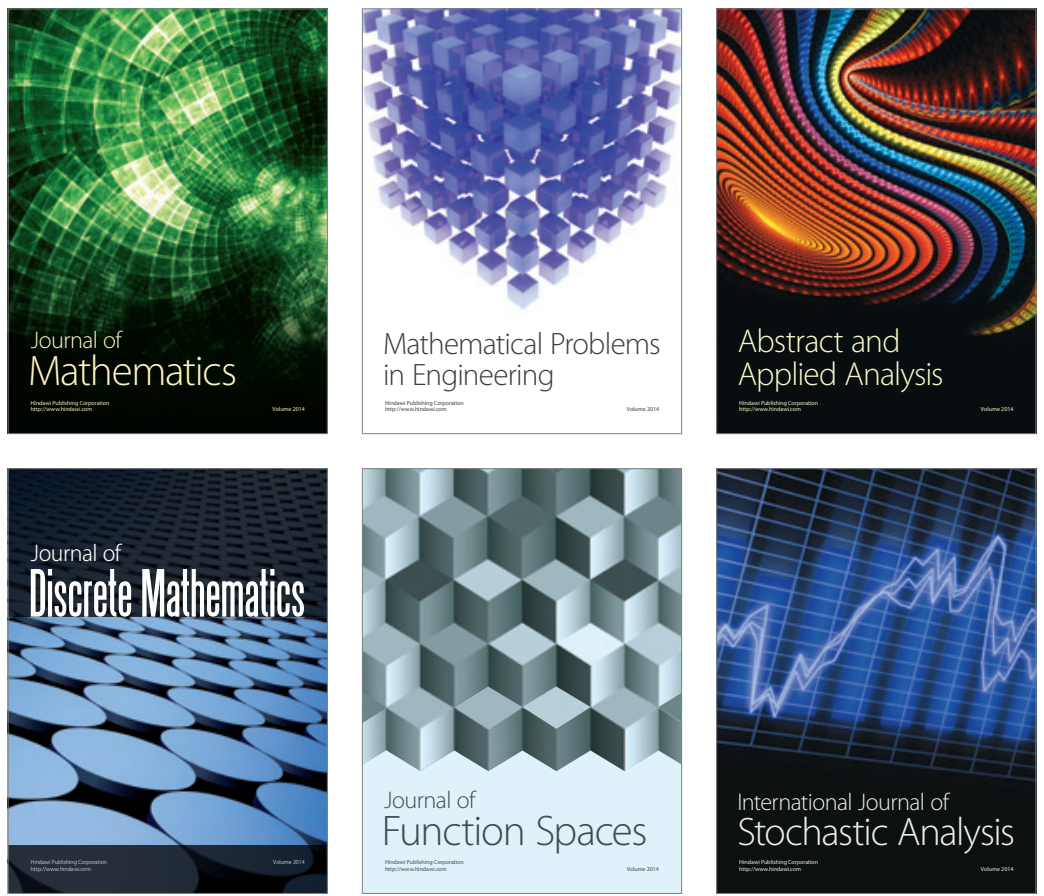

Journal of

Function Spaces

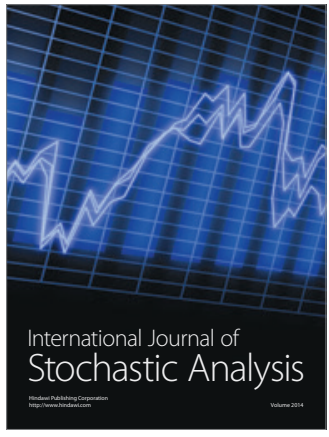

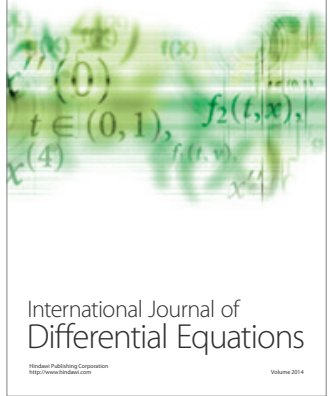
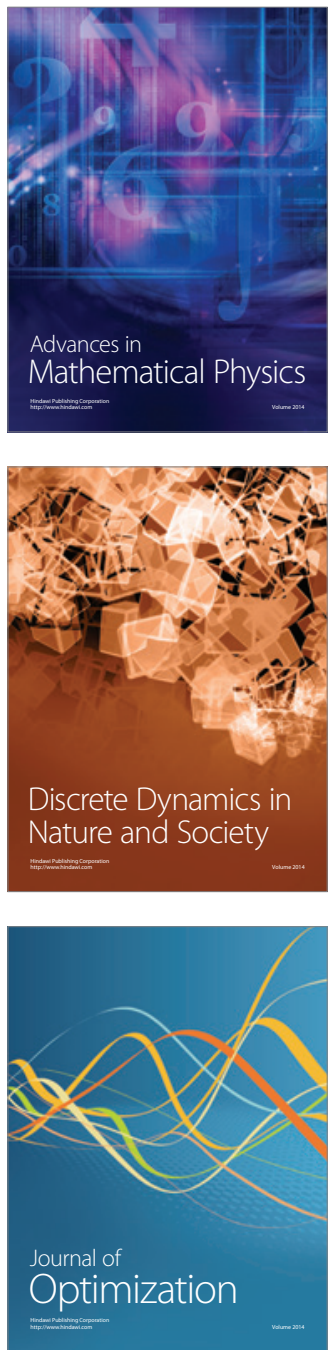